\title{
Two-phase flow equations with outflow boundary conditions in the hydrophobic-hydrophilic case
}

\author{
Michael Lenzinger and Ben Schweizer
}

Preprint 2008-12

Mai 2008

Fakultät für Mathematik

Technische Universität Dortmund

Vogelpothsweg 87

44227 Dortmund

$\underline{\text { tu-dortmund.de/MathPreprints }}$ 



\title{
Two-phase flow equations with outflow boundary conditions in the hydrophobic-hydrophilic case
}

\author{
Michael Lenzinger and Ben Schweizer ${ }^{1}$
}

20.5.2008

\begin{abstract}
We introduce an approximation procedure and provide existence results for two-phase flow equations in porous media. The medium can have hydrophobic and hydrophilic components such that the capillary pressure function is degenerate for extreme saturations. Our main interest is the outflow boundary condition which models an interface with open space. The approximate system introduces standard boundary conditions and can be used in numerical schemes. It allows the derivation of maximum principles. These are the basis for the derivation of the limiting system in the form of a variational inequality.
\end{abstract}

Keywords: two-phase flow, porous media, outflow boundary, maximum principle, variational inequality

\section{Introduction}

We study equations that describe two immiscible fluids in a porous medium, e.g. water and oil in rock, water and air in soil, or liquid water and water vapor in fuel cells. It is customary to model the porous medium with $\Omega \subset \mathbb{R}^{n}$, $n=2,3$, and to use the following two-phase flow equations on the time dependent domain $\Omega_{T}=\Omega \times(0, T)$.

$$
\begin{aligned}
\partial_{t} s & =\nabla \cdot\left(k_{1}(s) \nabla p_{1}\right) \\
-\partial_{t} s & =\nabla \cdot\left(k_{2}(s) \nabla p_{2}\right) \\
p_{1}-p_{2} & =p_{c}(s) .
\end{aligned}
$$

\footnotetext{
${ }^{1}$ Fakultät für Mathematik, TU Dortmund, Vogelpothsweg 87, 44227 Dortmund, Germany. michael.lenzinger@tu-dortmund.de and ben.schweizer@tu-dortmund.de
} 


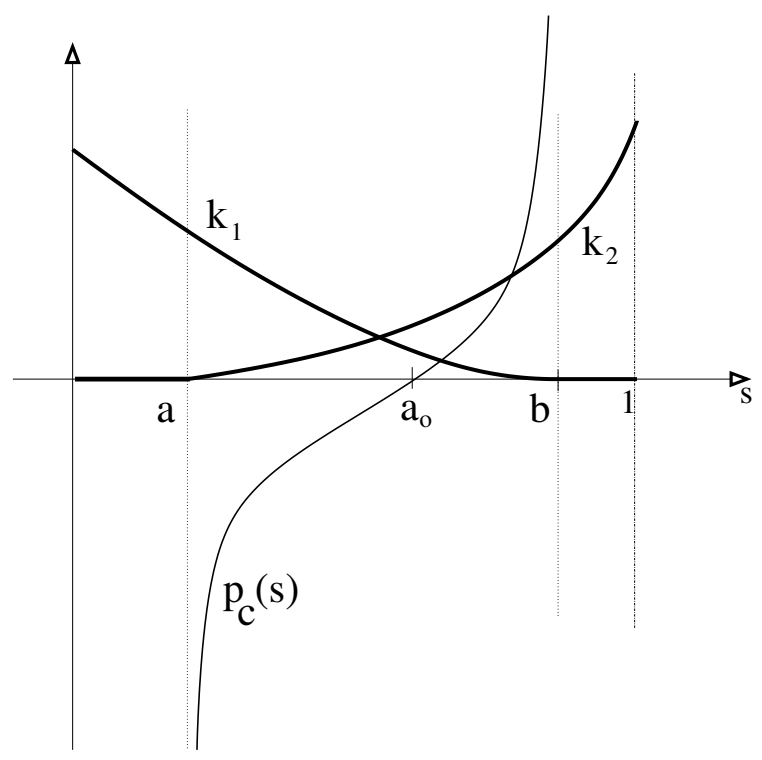

Figure 1: Typical shape of the saturation dependent functions $k_{j}$ and $p_{c}$. The permeability $k_{1}$ vanishes on $[0, a]$, the permeability $k_{2}$ on $[b, 1]$, the capillary pressure is monotonically increasing and unbounded for $s \searrow a$ and $s \nearrow b$.

Here $s_{1}:=s$ and $s_{2}:=1-s$ are the saturations of the two phases, defined as the volume fractions of pore space occupied by the fluids. The pressures $p_{j}$ are related to the phase velocities $v_{j}$ by Darcy's law, $v_{j}=-k_{j}(s) \nabla p_{j}$, $j=1,2$. Equations (1.1) and (1.2) are the laws of mass conservation for incompressible fluids, in the second equation appears $\partial_{t} s_{2}=-\partial_{t} s$. The permeabilities $k_{j}$ depend on the saturation and are typically degenerate. The capillary pressure function $p_{c}=p_{c}(s)$ depends on material properties of the involved substances and the microscopic properties of the medium. It relates the two pressures in (1.3). We remark that more general models can also be studied, of particular interest are hysteresis relations [5], [18]. Typical shapes of the coefficient functions $k_{j}(s)$ and $p_{c}(s)$ are depicted in Figure 1.

Three fundamental existence results are available for the degenerate system. In the case of Dirichlet and Neumann boundary conditions, in [9] a weak solution is constructed with a regularization of the permeabilities $k_{1}$ and $k_{2}$. Based on the global pressure formulation, [3] and [7] derive an existence result for the same boundary condition. Outflow conditions are treated in [1] in the case of hydrophilic media. A more detailed description of the literature is given at the end of the introduction.

We are interested in degenerate permeability functions $k_{j}$ such that $k_{1}(s)=$ 0 for $s \leq a$ and $k_{2}(s)=0$ for $s \geq b$. This reflects the physical fact that, e.g., isolated water reservoirs can be contained in the porous medium, but flow of 


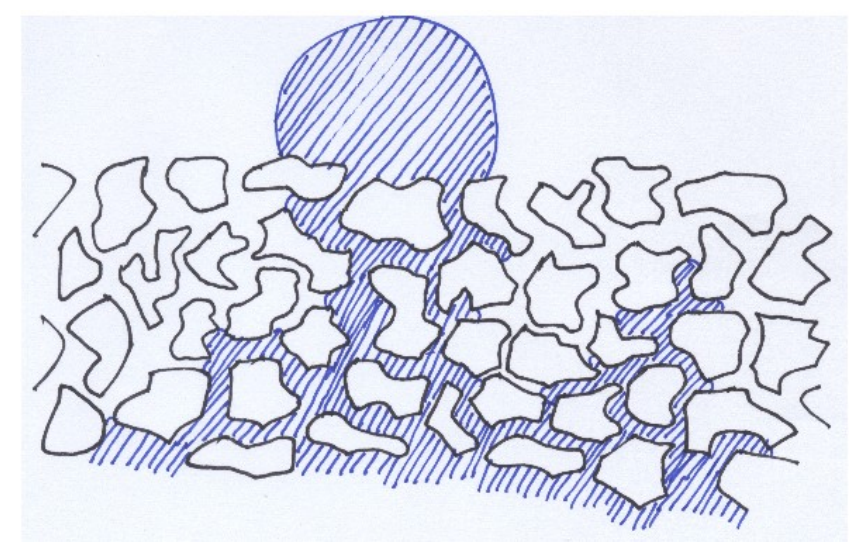

Figure 2: Illustration of a droplet formation on the outflow boundary. Due to a small curvature of the free boundary, the pressure difference between water and air is negligable in the drop. Therefore, as soon as outflow occurs, the capillary pressure vanishes along the boundary.

water is impossible since no connected pathways are available. In the case of materials with hydrophilic and hydrophobic parts, the absolute value of the capillary pressure may tend to infinity at the points $a$ and $b$. Thus, problem (1.1)-(1.3) is doubly degenerate.

\section{Boundary conditions}

A further difficulty for the modeling arises from the boundary conditions. The simplest case is that a layer of impenetrable material inhibits flow into and out of the porous volume. In this case, homogeneous Neumann conditions are appropriate. If, instead, the porous material is in contact with open space, filled with one of the two fluids, we have to deal with outflow (or inflow) conditions. We describe here briefly the droplet argument which motivates these outflow condition. In this description we assume that we study water (phase 1) and air (phase 2) in soil, and a boundary where the soil is in contact with open space, filled with air. For more detailed descriptions see [14] and the references therein.

The gas in the exterior has a constant pressure $p_{2}^{\text {out }}$. The gas phase inside the porous material is in contact with the gas in the exterior domain such that the pressures coincide. This leads to the Dirichlet condition $p_{2}=p_{2}^{\text {out }}$. For the water phase, we observe the following facts: No water can enter the porous medium from outside, since there is no water. Therefore $v_{1} \cdot n=$ $-k_{1}(s) \partial_{n} p_{1} \geq 0$. Let us now investigate the situation that water leaves the medium. In this case, water drops form on the surface of the porous material. 
Since their curvature is large in comparison to the curvature of interfaces inside the pores, the pressure difference between water and air is negligible at the boundary. This leads to the condition of a vanishing capillary pressure, $p_{1}-p_{2}=0$ if $v_{1} \cdot n>0$. Since water exits fast once that $p_{1}$ reaches $p_{2}^{\text {out }}$, this value can never be exceeded. Keeping in mind that one of the two conditions must be fulfilled, and with the normalization $p_{2}^{\text {out }}=0$, we find the outflow boundary condition

$$
\begin{aligned}
& p_{2}=0 \\
& v_{1} \cdot n \geq 0, \quad p_{1} \leq 0, \quad\left(v_{1} \cdot n\right) p_{1}=0 \quad \text { on } \Gamma_{\text {out }} .
\end{aligned}
$$

At the Dirichlet boundary $\Gamma_{D}$ we prescribe the pressure values, at the Neumann boundary $\Gamma_{N}$ the normal velocities.

$$
\begin{aligned}
& p_{1}=p_{1}^{D} \text { and } p_{2}=p_{2}^{D} \text { on } \Gamma_{D}, \\
& v_{1} \cdot n=0 \text { and } v_{2} \cdot n=0 \text { on } \Gamma_{N} .
\end{aligned}
$$

\section{Regularized equations and main results}

In the following we present a regularization of the above system with a parameter $\delta>0$. We introduce a sequence of strictly positive coefficient functions $k_{j}^{\delta}, j=1,2$, and bounded capillary pressure functions $p_{c}^{\delta}$, such that system (1.1)-(1.3) tranforms into a non-degenerate problem.

We furthermore discretize the time variable $t$. We introduce a small number $h>0$ such that $T=h N$ for $N \in \mathbb{N}$ and introduce the discrete time steps $t_{k}=k h, k=0,1, \ldots, N$. We denote the time discrete solution at time $t_{k}$ with $s_{k}^{h}, p_{1, k}^{h}, p_{2, k}^{h}$ and recall that the solutions depend additionally on $\delta$.

$$
\begin{aligned}
\frac{s_{k}^{h}-s_{k-1}^{h}}{h} & =\nabla \cdot\left(k_{1}^{\delta}\left(s_{k}^{h}\right) \nabla p_{1, k}^{h}\right) \\
-\frac{s_{k}^{h}-s_{k-1}^{h}}{h} & =\nabla \cdot\left(k_{2}^{\delta}\left(s_{k}^{h}\right) \nabla p_{2, k}^{h}\right) \\
p_{1, k}^{h}-p_{2, k}^{h} & =p_{c}^{\delta}\left(s_{k}^{h}\right)
\end{aligned}
$$

with some initial condition $s_{0}^{h}=s_{0}$ in $\Omega$.

We furthermore regularize the outflow boundary condition (1.4). For phase 1 , we relate the normal velocity to the positive part of the pressure,

$$
\begin{aligned}
p_{2, k}^{h} & =0 \\
n \cdot \nabla p_{1, k}^{h} & =-\frac{1}{\delta}\left(p_{1, k}^{h}\right)_{+} \quad \text { on } \Gamma_{\text {out }} .
\end{aligned}
$$


This regularization guarantees that the normal velocity is always positve. Furthermore, a positive pressure results in a fast outflow. For $\delta \rightarrow 0$, this effect limits the pressure and yields, eventually, condition (1.4). The remaining boundary conditions need not be regularized. We keep

$$
\begin{aligned}
& p_{1, k}^{h}=p_{1}^{D}\left(t_{k}\right) \text { and } p_{2, k}^{h}=p_{2}^{D}\left(t_{k}\right) \text { on } \Gamma_{D}, \\
& n \cdot \nabla p_{1, k}^{h}=0 \text { and } n \cdot \nabla p_{2, k}^{h}=0 \text { on } \Gamma_{N} .
\end{aligned}
$$

\section{Main results}

In our first result, we study the regularized equations (1.6)-(1.10). We prove the existence of solutions and derive bounds that are independent of $\delta$ and $h$. The main ingredient is a maximum principle. Such a maximum principle seems intuitive, in particular if one keeps in mind that the global pressure (see (2.1)) satisfies an elliptic equation. But proofs turn out to be intricate since no boundary condition allows to control the global pressure at the outflow boundary. In our second result we perform the limit $\delta, h \rightarrow 0$ and find solutions $\left(s, p_{1}, p_{2}\right)$ of the original degenerate problem. In this step we must use a variational formulation for the outflow boundary condition (1.4).

We now specify the general assumptions on the data. The porous medium is given by the domain $\Omega \subset \mathbb{R}^{n}$. In order to have regularity results at our disposal, we restrict to the case of parallelepipeds $\Omega=\left(a_{1}, b_{1}\right) \times \ldots \times\left(a_{n}, b_{n}\right)$ with $n \geq 2$. We assume that the left boundary is the Dirichlet boundary, $\Gamma_{D}=\left\{a_{1}\right\} \times\left(a_{2}, b_{2}\right) \times\left(a_{3}, b_{3}\right)$, the right boundary is the outflow boundary, $\Gamma_{\text {out }}=\left\{b_{1}\right\} \times\left(a_{2}, b_{2}\right) \times\left(a_{3}, b_{3}\right)$, the remainder consist of impenetrable walls, $\Gamma_{N}=\partial \Omega \backslash\left(\bar{\Gamma}_{D} \cup \bar{\Gamma}_{\text {out }}\right)$. For some $T>0$, the time cylinder is $\Omega_{T}:=\Omega \times$ $(0, T)$. The inflow data are given by functions $p_{j}^{D}: \bar{\Omega}_{T} \rightarrow \mathbb{R}$ with $p_{j}^{D} \in$ $C^{1}\left([0, T], C^{2, \alpha}(\bar{\Omega}) \cap H^{1}(\Omega)\right)$ satisfying $p_{j}^{D}=0$ on $\Gamma_{\text {out }}, p_{2}^{D} \geq 0$ in $\Omega_{T}$. The initial saturation is $s_{0} \in C^{\alpha}(\bar{\Omega})$ with $a<s_{0}<b$ on $\Omega$. Finally, we assume the compatibility $p_{c}\left(s_{0}\right)=p_{1}^{D}(., 0)-p_{2}^{D}(., 0)$ on $\Gamma_{D}$.

Theorem 1 (Bounds for the saturation). Let the coefficient functions satisfy Assumption 2.1. Then, for every $\delta>0$, there exists a weak solution to (1.6)-(1.10). Furthermore, for some $\delta_{0}>0$, there exist constants a $<$ $S_{\min } \leq S_{\max }<b$ and $C>0$ such that, for all $0<\delta \leq \delta_{0}$ and all $k \leq N$

1. The saturation $s_{k}^{h}$ is bounded in $\bar{\Omega}_{T}$,

$$
S_{\min } \leq s_{k}^{h} \leq S_{\max } \quad \text { in } \bar{\Omega}_{T},
$$

2. The pressure functions are bounded in $\bar{\Omega}_{T}$,

$$
\max _{\bar{\Omega}_{T}}\left\{\left|p_{1, k}^{h}\right|+\left|p_{2, k}^{h}\right|\right\} \leq C .
$$


Theorem 2 (Regularization limit). Let Assumptions 2.1 hold. For a subsequence $(\delta, h) \rightarrow 0$, the regularized solutions $\left(s^{h}, p_{1}^{h}, p_{2}^{h}\right)$ converge weakly in $L^{2}\left(0, T, H^{1}(\Omega)\right)$ to limit functions $\left(s, p_{1}, p_{2}\right)$ that satisfy

$$
\begin{aligned}
\partial_{t} s-\nabla \cdot\left(k_{1}(s) \nabla p_{1}\right) & =0 \text { in } \mathcal{D}^{\prime}\left(\Omega_{T}\right), \\
-\partial_{t} s-\nabla \cdot\left(k_{1}(s) \nabla p_{2}\right) & =0 \text { in } \mathcal{D}^{\prime}\left(\Omega_{T}\right), \\
p_{1}-p_{2} & =p_{c}(s(.)) \text { a.e. in } \Omega_{T} .
\end{aligned}
$$

The standard boundary conditions (1.5) hold in the sense of traces. At the outflow boundary we have $p_{2}=0, p_{1} \leq 0$ in the sense of traces and $v_{1} \cdot n \geq 0$ in the distributional sense. Additionally, for a.e. $t \in(0, T)$ there holds the variational inequality

$$
\begin{aligned}
& -\int_{\Omega}\left(P_{c}(s(t))-P_{c}\left(s^{0}\right)\right)+\int_{\Omega} s(t)\left(\phi_{1}-\phi_{2}\right)(t)-\int_{\Omega} s^{0}\left(\phi_{1}-\phi_{2}\right)(0) \\
& -\int_{\Omega_{t}} s \partial_{t}\left(\phi_{1}-\phi_{2}\right)-\sum_{j} \int_{\Omega_{t}} k_{j}(s)\left|\nabla p_{j}\right|^{2}+\sum_{j} \int_{\Omega_{t}} k_{j}(s) \nabla p_{j} \nabla \phi_{j} \geq 0
\end{aligned}
$$

for all $\phi_{j} \in C^{1}\left(\bar{\Omega}_{T}\right), \phi_{j}=p_{j}^{D}$ on $\Gamma^{D}, \phi_{1} \leq 0$ and $\phi_{2}=0$ on $\Gamma_{\text {out }}$.

We will prove Theorem 1 in Section 2 and Theorem 2 in Section 3.

Remark (variational outflow condition). The variational inequality (1.16) was introduced in [1] to define weak solutions for the outflow problem.

Concerning the equivalence of the original system and the equations of Theorem 2 we note the following. Every strong solution of the original system (1.1)-(1.5) is also a solution in the sense of Theorem 2. The variational inequality (1.16) follows with the test function $\varphi_{j}=p_{j}-\phi_{j}$.

Vice versa, every sufficiently regular solution of the variational inequality (1.16) with $p_{2}=0$ and $p_{1} \leq 0$ on $\Gamma_{\text {out }}$ is a solution of the original system. The distributional limit equations can be obtained from inequality (1.16) by inserting $\phi_{j}=p_{j}+\varphi_{j}$ with arbitrary $\varphi_{j} \in C_{0}^{\infty}\left(\Omega_{T}\right)$. At the outflow boundary one finds

$$
\int_{\Gamma_{\text {out }}} v_{1} \cdot n\left(p_{1}-\phi_{1}\right) \geq 0 \quad \forall \phi_{1} \leq 0,
$$

which implies the outflow conditions (1.4). For further considerations we refer to [1], Lemma 2.7.

Possible generalizations. Many of the assumptions are made to have

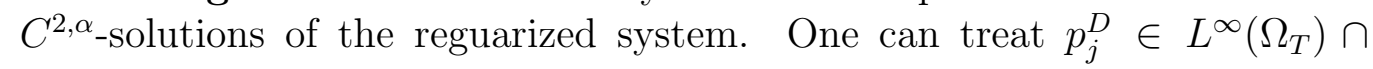


$L^{\infty}\left((0, T), H^{1}(\Omega)\right)$ and $s_{0} \in L^{\infty}(\Omega)$ with $a<s_{0}<b$ a.e. on $\Omega$ with one further regularization procedure.

Also more general domains can be treated. The problem are the contact lines $\bar{\Gamma}_{D} \cap \bar{\Gamma}_{N}$ and $\bar{\Gamma}_{\text {out }} \cap \bar{\Gamma}_{N}$, where different boundary conditions meet. At these lines, in general, the time discrete solutions are not $C^{1}$ and our maximum principle can not be applied. But it is possible to introduce a regularization of the domain and to approximate a general domain with one where different boundary conditions meet only at interfaces that meet at a $90^{\circ}$ angle. The essential condition is $\bar{\Gamma}_{D} \cap \bar{\Gamma}_{\text {out }}=\emptyset$, which is also a physical constraint. We regard also $p_{2}^{D} \geq 0$ as a physical constraint; it guarantees that no gas exits at $\Gamma_{D}$. In the opposite case one should use an inflow condition rather than a Dirichlet condition on $\Gamma_{D}$.

\section{Comparison to the literature}

The one-phase flow equation or Richards' equation with outflow boundary condition and degenerate coefficient functions is studied e.g. in [2] and [19]. The existence result of [2] is based on a time discretization which is, in itself, formulated with variational inequalities. Instead, [19] uses a stronger solution concept which allows a more direct formulation of the outflow condition with a defect measure. Approximate solutions are defined with a $\delta$-regularization, in particular, the regularized outflow relation (1.9) is introduced. Our contribution combines both concepts in order to treat the two-phase flow system. The regularization is as in [19], but the outflow condition can only be derived as a variational inequality. The time discretization serves for two purpuses: It allows to find smooth solutions in order to derive maximum principles by classical means. Additionally, it allows the strong formulation of the nonlinear regularized outflow condition in (1.9).

The two-phase flow equations are studied in the fundamental contribution of Alt and DiBenedetto [1]. The aproach is much like that of [2], with a time discretization, transformed pressures, and the use of variational inequalities. The proofs are technically more involved since e.g. a vanishing saturation is not excluded. We note that only the hydrophilic case is studied in [1]. The authors assume that: (i) $p_{c}$ is strictly monotone to define $s_{i}=s_{i}\left(p_{1}-p_{2}\right)$ between equations (1.1) and (1.2), (ii) $p_{c} \leq p_{\max }$ in (1.3), (iii) $v_{1} \neq 0$ only if $p_{c}=p_{\text {max }}$ in (1.6). Our interest here is outflow at capillary pressure 0, even though positive capillary pressures are possible.

Dirichlet- and Neumann-boundary conditions are studied in [9], where, once more, the permeability functions $k_{1}$ and $k_{2}$ are approximated by nondegenerate versions. The main tool in that work are maximum principles which guarantee that the regularized saturations remain bounded away from 
the degenerate points. Our aim is to treat the outflow boundary condition along the same lines. Our first step is to establish bounds for the regularized saturation and for the regularized pressure. These bounds allow to perform the limit procedure much as in [9]. While in [9] testing procedures are used to derive the estimates, the outflow condition forces us to use geometrical techniques and to work with classical solutions.

Other existence results for the two-phase flow system can be found in $[3,7]$, regularity results in [8], e.g. Hölder continuity of the saturation. These contributions are based on the global pressure formulation of the problem, exploiting that the global pressure solves a non-degenerate elliptic equation. Accordingly, only Dirichlet- and Neumann conditions are studied, since outflow boundaries admit no boundary condition for the global pressure. We mention at this point that we nevertheless use the global pressure as an auxiliary variable in our derivation of the uniform bounds.

Regarding the interesting field of homogenization of the two-phase flow system we mention $[4,6,20]$, regarding numerical approaches see $[11,13,16]$, for fuel cell applications we refer to $[12,15]$ and the references therein. [16] contains also a maximum principle for standard boundary conditions.

\section{Uniform bounds}

Aim of this section are bounds on the saturation and the pressures, bounds that are independent of $\delta>0$ and $h>0$. Typical coefficient functions are illustrated in Figure 1, the precise assumptions are the following.

Assumption 2.1 (Assumptions on the coefficients). We assume that the coefficients and their regularizations satisfy, for points $0 \leq a<a_{0}<b \leq 1$ and constants $c_{0}, c_{1}, K>0$ :

(i) Degenerate coefficient functions. The permeabilities $k_{j} \in C^{0}([0,1])$ satisfy $0 \leq k_{j} \leq K, k_{1}+k_{2} \geq c_{0}>0$, with $k_{1}(s)=0 \Leftrightarrow s \in[0, a]$ and $k_{2}(s)=0 \Leftrightarrow s \in[b, 1]$. The capillary pressure $p_{c} \in C^{2,1}((a, b), \mathbb{R})$ satisfies $p_{c}\left(a_{0}\right)=0, \partial_{s} p_{c} \geq c_{1}>0$, and $\left|p_{c}(s)\right| \rightarrow \infty$ as $s \rightarrow a$ or $s \rightarrow b$.

(ii) Regularized coefficient functions. The regularized permeabilities $k_{j}^{\delta} \in$ $C^{1,1}([0,1])$ satisfy $k_{j}^{\delta} \geq k_{j}$ and $0<c_{\delta} \leq k_{j}^{\delta} \leq 2 K$ with $c_{\delta}$ depending on $\delta$. The capillary pressure function is regularized to $p_{c}^{\delta} \in C^{2,1}((0,1))$ with $\partial_{s} p_{c}^{\delta} \geq c_{1}>0$ and $\left|p_{c}^{\delta}(s)\right| \rightarrow \infty$ for $s \rightarrow 0$ or $s \rightarrow 1$.

(iii) Convergence of the regularized coefficients. There holds $k_{j}^{\delta} \searrow k_{j}$ uniformly on $[0,1]$. For every compact interval $I \subset(a, b)$ there exists $\delta_{0}=\delta_{0}(I)$ such that $p_{c}^{\delta}=p_{c}$ on $I$ for all $\delta \leq \delta_{0}$. 
We will use the global pressure as e.g. [7]. It is defined as

$$
p_{g}^{\delta}:=p_{2}^{\delta}+\int_{a_{0}}^{s} k^{\delta}(\xi) \partial_{s} p_{c}^{\delta}(\xi) d \xi, \quad k^{\delta}:=\frac{k_{1}^{\delta}}{k_{1}^{\delta}+k_{2}^{\delta}} .
$$

We recall that $a_{0}$ is the saturation with vanishing capillary pressure, i.e. $p_{c}\left(a_{0}\right)=0$. Our maximum principle exploits the fact that the global pressure solves the elliptic equation

$$
\nabla \cdot\left(\left[k_{1}^{\delta}(s)+k_{2}^{\delta}(s)\right] \nabla p_{g}^{\delta}\right)=0 .
$$

For ease of notation we omit in the following the superscript $\delta$.

\subsection{Existence and energy estimates}

For the derivation of a priori estimates it is convenient to work with interpolations of the time discrete solutions. For every $k=0,1, \ldots, N-1$ and every $t \in\left(t_{k}, t_{k+1}\right], t=\mu t_{k}+(1-\mu) t_{k+1}$, we define the piecewise constant and the piecewise affine interpolation as

$$
\begin{aligned}
& \bar{s}^{h}(t)=s_{k+1}^{h} \\
& \hat{s}^{h}(t)=\mu s_{k}^{h}+(1-\mu) s_{k+1}^{h} .
\end{aligned}
$$

and in the same way we define $\bar{p}_{j}^{h}$ and $\hat{p}_{j}^{h}$. Furthermore, we denote by $p_{j}^{D, h}$ the constant interpolation of $p_{j}^{D}\left(t_{k}\right)$. For the a priori estimates we define the inverse of $p_{c}$ and the primitives

$$
\theta^{\delta}=\left(p_{c}^{\delta}\right)^{-1}: \mathbb{R} \rightarrow(0,1), \quad \Theta^{\delta}(\rho)=\int_{0}^{\rho} \theta^{\delta}(\xi) d \xi, \quad P_{c}^{\delta}(\sigma)=\int_{a_{0}}^{\sigma} p_{c}^{\delta}(\zeta) d \zeta .
$$

Lemma 2.2 (The time discrete regularized system). There exists a weak solution $s_{k}^{h}, p_{j, k}^{h}$ of time discrete system (1.6)-(1.10). The interpolations $\bar{s}^{h}, \bar{p}_{j}^{h}$ satisfy the following estimates with a constant $C>0$ independent of $\delta>0$ and $h>0$.

$$
\begin{array}{r}
\int_{\Omega_{T}}\left(k_{1}^{\delta}\left|\nabla \bar{p}_{1}^{h}\right|^{2}+k_{2}^{\delta}\left|\nabla \bar{p}_{2}^{h}\right|^{2}\right) \leq C, \\
\int_{\Omega_{T}} k_{1}^{\delta} k_{2}^{\delta}\left(\partial_{s} p_{c}^{\delta}\right)^{2}\left|\nabla \bar{s}^{h}\right|^{2} \leq C, \\
\sup _{[0, T]} \int_{\Omega} P_{c}^{\delta}\left(\hat{s}^{h}(\cdot, t)\right) \leq C .
\end{array}
$$

The estimates hold also for the linear interpolations $\hat{s}^{h}$ and $\hat{p}_{j}^{h}$. Every solution has the regularity

$$
s_{k}^{h}, p_{j, k}^{h} \in C^{2, \alpha}(\bar{\Omega}) .
$$


Proof. The proof is straight-forward and sketched here to highlight the essential steps.

Existence. A solution for the single time step can be constructed as the fixed point of a map $T: s^{\text {old }} \mapsto s^{\text {new }}$, where $s^{\text {new }}$ is a solution of problem (1.6)-(1.8) with fixed coefficients $k_{j}^{\delta}\left(s^{\text {old }}\right)$. The elliptic system for $\left(p_{1}, p_{2}\right)$ and $s^{\text {new }}=\theta^{\delta}\left(p_{1}-p_{2}\right)$ can be solved with the variational formulation

$$
\begin{aligned}
\inf _{\left(\varphi_{1}, \varphi_{2}\right)} \int_{\Omega}\left\{\frac{1}{2} k_{1}^{\delta}\left(s^{\text {old }}\right)\left|\nabla \varphi_{1}\right|^{2}+\frac{1}{2} k_{2}^{\delta}\left(s^{\text {old }}\right)\left|\nabla \varphi_{2}\right|^{2}\right. & \\
& \left.+\frac{1}{h} \Theta^{\delta}\left(\varphi_{1}-\varphi_{2}\right)-\frac{s_{k-1}^{h}}{h} \varphi_{1}+\frac{s_{k-1}^{h}}{h} \varphi_{2}\right\} .
\end{aligned}
$$

Schauder's fixed point theorem provides the existence of $s$ with $T(s)=s$ in an appropriate ball.

A priori estimates in the time dependent problem. The estimates are obtained by testing (1.6) with $\left(\bar{p}_{1}^{h}-p_{1}^{D, h}\right)$, testing (1.7) with $\left(\bar{p}_{2}^{h}-p_{2}^{D, h}\right)$ and adding. On the left-hand side we have

$$
\begin{aligned}
A:= & \int_{0}^{T} \int_{\Omega} \partial_{t} \hat{s}^{h} p_{c}^{\delta}\left(\bar{s}^{h}\right)-\int_{0}^{T} \int_{\Omega} \partial_{t} \hat{s}^{h} p_{c}^{\delta}\left(s^{D, h}\right) \\
= & \int_{0}^{T} \int_{\Omega} \partial_{t} P_{c}^{\delta}\left(\hat{s}^{h}\right)+\sum_{k=0}^{N-1} \int_{t_{k}}^{t_{k+1}} \int_{\Omega} \frac{s_{k+1}^{h}-s_{k}^{h}}{h}\left(p_{c}^{\delta}\left(\bar{s}^{h}\right)-p_{c}^{\delta}\left(\hat{s}^{h}\right)\right) \\
& -\int_{0}^{T} \int_{\Omega} \partial_{t} \hat{s}^{h} p_{c}^{\delta}\left(s^{D, h}\right) \\
\geq & \int_{\Omega} P_{c}^{\delta}\left(\hat{s}^{h}\right)(T)-\int_{\Omega} P_{c}^{\delta}\left(s_{0}\right)-C .
\end{aligned}
$$

Here, we used the monotonicity of the capillary pressure $p_{c}^{\delta}$ and the boundedness of $p_{j}^{D}$ and its time derivatives.

Comparing with the right-hand side yields

$$
\begin{aligned}
A= & -\int_{\Omega_{T}}\left(k_{1}^{\delta}\left|\nabla \bar{p}_{1}^{h}\right|^{2}+k_{2}^{\delta}\left|\nabla \bar{p}_{2}^{h}\right|^{2}\right)-\int_{0}^{T} \int_{\Gamma_{\text {out }}} \frac{k_{1}^{\delta}\left(\bar{s}^{h}\right)}{\delta}\left(\bar{p}_{1}^{h}\right)_{+} \bar{p}_{1}^{h} \\
& +\int_{\Omega_{T}} k_{1}^{\delta} \nabla \bar{p}_{1}^{h} \nabla p_{1}^{D, h}+k_{2}^{\delta} \nabla \bar{p}_{2}^{h} \nabla p_{2}^{D, h} .
\end{aligned}
$$

This yields (2.5) and (2.7), since the boundary integral is non-negative. The estimate (2.6) for the saturation follows from (2.5). 
Regularity. For any weak solution we consider, in a first step, (1.6) and (1.7) as linear elliptic equations for $p_{1, k}^{h}$ and $p_{2, k}^{h}$ with bounded data $f=$ $\pm\left(s_{k}^{h}-s_{k-1}^{h}\right) / h$. Regularity theory implies the Hölder continuity of the pressures, $p_{j, n}^{h} \in C^{\alpha}(\bar{\Omega})$. This result can be found e.g. in [10], Chapter 3, Theorem 14.1 for Dirichlet boundary data.

The improved regularity and relation (1.8) provide the Hölder continuity of $s_{k}^{h}$. Invoking again regularity theory, now with Hölder continuous coefficients, we conclude that $p_{j, k}^{h}$ have Hölder continuous second derivates in $\Omega$. The $C^{2, \alpha}$ regularity holds also for $s_{k}^{h}$ due to smoothness of $p_{c}$.

Concerning the boundary we note the following. The $C^{2, \alpha}$ regularity holds up to smooth Dirichlet boundaries, see [10], Chapter 3, Theorem 1.1. Again, we use it also for smooth boundaries with an inhomogeneous Neumann condition, i.e. on $\Gamma_{\text {out }}$. In our situation of parallelepipeds $\Omega$ we can use a reflection principle and extend the solution as even functions across Neumann boundaries (compare e.g. [17]). In this way, points on the Neumann boundary become inner points and the $C^{2, \alpha}$-estimates remain valid on $\bar{\Omega}$. Furthermore, points on $\bar{\Gamma}_{D} \cap \bar{\Gamma}_{N}$ and $\bar{\Gamma}_{\text {out }} \cap \bar{\Gamma}_{N}$ can be regarded as inner points of $\Gamma_{D}$ and $\Gamma_{\text {out }}$, respectively.

\subsection{Maximum principles}

For the time discrete solution we now show that saturation and global pressure take their maxima and minima at inflow or outflow boundaries.

The saturation. We show that $s^{h}$ attains its maximal and minimal value on the parabolic boundary of the domain $\Omega_{T}$. We set $\Sigma_{0}=\Omega \times\{0\}, \Gamma_{0}=$ $\Gamma_{D} \cup \Gamma_{\text {out }}$, and $\Gamma_{0, t}=\Gamma_{0} \times(0, t)$.

Lemma 2.3 (Maximum principle for the saturation). Every solution $\bar{s}^{h}, \bar{p}_{1}^{h}, \bar{p}_{2}^{h}$ of (1.6)-(1.10) satisfies the maximum principle

$$
\max _{\bar{\Omega}_{t}} \bar{s}^{h} \leq \max _{\bar{\Sigma}_{0} \cup \bar{\Gamma}_{0, t}} \bar{s}^{h}
$$

for all $0 \leq t \leq T$. The analogous result holds for the minimum of $s$ :

$$
\min _{\bar{\Omega}_{t}} \bar{s}^{h} \geq \min _{\bar{\Sigma}_{0} \cup \bar{\Gamma}_{0, t}} \bar{s}^{h} .
$$

Proof. We argue by contradiction. Let $k$ be the smallest positive integer such that (2.9) fails for $t=t_{k}$. We find a point $x_{0} \in \bar{\Omega}$ such that $s_{k}^{h}$ has its maximum in $x_{0}$. By assumption, $x_{0} \notin \bar{\Gamma}_{0}$. We recall that, due to the reflection principle, points on the Neumann boundary can be regarded as inner points. It is therefore sufficient to consider the case $x_{0} \in \Omega$. 
Since $x_{0}$ is a maximum, $\nabla s_{k}^{h}\left(x_{0}\right)=0$, such that equation (1.6) yields

$$
k_{1}\left(s_{k}^{h}\right) \Delta p_{1, k}^{h}\left(x_{0}\right)=\frac{s_{k}^{h}\left(x_{0}\right)-s_{k-1}^{h}\left(x_{0}\right)}{h}>0 .
$$

Analogously, (1.7) provides

$$
k_{2}\left(s_{k}^{h}\right) \Delta p_{2, k}^{h}\left(x_{0}\right)<0 .
$$

The capillary pressure $p_{c}$ is a strictly increasing function of the saturation $s$ and therefore also has an interior maximum at the point $\left(x_{0}, t_{k}\right)$. We therefore have

$$
0<\Delta p_{1, k}^{h}\left(x_{0}\right)-\Delta p_{2, k}^{h}\left(x_{0}\right)=\Delta\left[p_{c}\left(s_{k}^{h}\right)\right]\left(x_{0}\right) \leq 0,
$$

the desired contradiction.

The same argument provides the result for minima.

The global pressure. The global pressure in the time discrete setting is

$$
p_{g, k}^{h}:=p_{2, k}^{h}+\int_{a_{0}}^{s_{k}^{h}} k^{\delta}(\xi) \partial_{s} p_{c}^{\delta}(\xi) d \xi, \quad k^{\delta}:=\frac{k_{1}^{\delta}}{k_{1}^{\delta}+k_{2}^{\delta}} .
$$

Just as in the time continuous case, the global pressure satisfies an elliptic equation. Indeed, (1.6)-(1.8) imply

$$
\nabla \cdot\left(\left[k_{1}^{\delta}\left(s_{k}^{h}\right)+k_{2}^{\delta}\left(s_{k}^{h}\right)\right] \nabla p_{g, k}^{h}\right)=0 .
$$

Lemma 2.4 (Maximum principle for the global pressure). For every time discrete solution, the global pressure satisfies an elliptic maximum principle. There holds

$$
\min _{\bar{\Omega}} p_{g, k}^{h} \geq \min _{\bar{\Gamma}_{0}} p_{g, k}^{h}, \quad \max _{\bar{\Omega}} p_{g, k}^{h} \leq \max _{\bar{\Gamma}_{0}} p_{g, k}^{h}
$$

for every $k=1, \ldots, N$.

Proof. The elliptic equation (2.12) implies that extrema of the global pressure lie on the boundary. For extrema on the boundary $\Gamma_{N}$ holds

$$
\nabla p_{g, k}^{h} \cdot n=\nabla p_{2, k}^{h} \cdot n+k^{\delta}\left(s_{k}^{h}\right) \nabla\left(p_{1, k}^{h}-p_{2, k}^{h}\right) \cdot n=0,
$$

in contradiction to the Lemma of Hopf. 


\subsection{Uniform bounds}

Uniform bounds for pressure and saturation are derived with a detailed analysis of the maxima and minima of the saturation. Due to the maximum principle of Lemma 2.3, we can assume that, for $\left(x_{M}, t_{M}\right) \in \bar{\Gamma}_{0, T}$ and $\left(x_{m}, t_{m}\right) \in \bar{\Gamma}_{0, T}$

$$
\begin{gathered}
\bar{s}^{h}\left(x_{M}, t_{M}\right)=M:=\max _{\bar{\Omega}_{T}} \bar{s}^{h}, \\
\bar{s}^{h}\left(x_{m}, t_{m}\right)=m:=\min _{\bar{\Omega}_{T}} \bar{s}^{h} .
\end{gathered}
$$

By the strict monotonicity of the capillary pressure we also have the global maximum and minimum of $p_{c}^{\delta}\left(\bar{s}^{h}\right)=\bar{p}_{1}^{h}-\bar{p}_{2}^{h}$ at the points $\left(x_{M}, t_{M}\right)$ and $\left(x_{m}, t_{m}\right)$, respectively.

Upper bound for the saturation. Starting from the Dirichlet data, we introduce numbers that will provide the uniform bounds. On the Dirichlet boundary, $p_{c}$ and $p_{2}$ are bounded by

$$
p_{c, \text { max }}:=\max \left\{0, \max _{\bar{\Gamma}_{D} \times[0, T]}\left(p_{1}^{D}-p_{2}^{D}\right)\right\}, \quad p_{2, \max }:=\max _{\bar{\Gamma}_{D} \times[0, T]} p_{2}^{D} \geq 0,
$$

which allows to define the numbers

$$
\begin{aligned}
p_{\text {max }} & :=p_{2, \text { max }}+p_{c, \text { max }}, \\
s_{c, \text { max }} & :=p_{c}^{-1}\left(p_{c, \text { max }}\right), \quad s_{\max }:=p_{c}^{-1}\left(\frac{4 K}{k_{1}\left(a_{0}\right)} p_{\max }\right)>s_{c, \text { max }} .
\end{aligned}
$$

The initial saturation is bounded by $s_{0, \max }:=\max _{\bar{\Omega}} s_{0}$.

Lemma 2.5. There exists $\delta_{0}>0$ such that, for all $\delta \leq \delta_{0}$, the saturation is bounded from above,

$$
\max _{\bar{\Omega}_{T}} \bar{s}^{h} \leq S_{\text {max }}:=\max \left(s_{\max }, s_{0, \max }\right) .
$$

Proof. We choose $I \subset(a, b)$ with $s_{\max }, s_{0, \max }, s_{c, \max } \in I$ and then $\delta_{0}>0$ sufficiently small to have $p_{c}^{\delta}=p_{c}$ on $I$ for all $\delta \leq \delta_{0}$. For a contradiction argument, let us assume that $M>S_{\max }$. By choice of $S_{\max }$ and the maximum principle for $s$ in Lemma 2.3, we necessarily have $x_{M} \in \bar{\Gamma}_{\text {out }}$.

Along the Dirichlet boundary, the global pressure satisfies

$$
\begin{aligned}
\max _{\bar{\Gamma}_{D}} \bar{p}_{g}^{h}\left(\cdot, t_{M}\right) & \leq p_{2, \max }+\int_{a_{0}}^{s_{c, \max }} k^{\delta} \partial_{s} p_{c}^{\delta} \\
& \leq p_{2, \max }+\int_{a_{0}}^{s_{c, \max }} \partial_{s} p_{c}=p_{\max } .
\end{aligned}
$$


On the other hand, in the maximum with saturation $M>S_{\max }$

$$
\bar{p}_{g}^{h}\left(x_{M}, t_{M}\right)=\int_{a_{0}}^{M} k^{\delta} \partial_{s} p_{c}^{\delta} \geq \frac{1}{4 K} \int_{a_{0}}^{M} k_{1}^{\delta} \partial_{s} p_{c}^{\delta} \geq \frac{k_{1}^{\delta}\left(a_{0}\right)}{4 K} p_{c}^{\delta}(M)>p_{\max },
$$

since $p_{c}^{\delta}(M)>p_{c}^{\delta}\left(s_{\max }\right)=p_{c}\left(s_{\max }\right)$ and $k_{1}^{\delta}\left(a_{0}\right)>k_{1}\left(a_{0}\right)$ for all $\delta \leq \delta_{0}$ by construction. We find that $x_{M}$ is not on the Dirichlet boundary. With the maximum principle for the global pressure of Lemma 2.4 we conclude that, at time $t=t_{M}$, also the global pressure $\bar{p}_{g}^{h}$ must attain its global maximum on the outflow boundary $\bar{\Gamma}_{\text {out }}$.

Since $\bar{p}_{2}^{h}=0$ on $\Gamma_{\text {out }}$, saturation and global pressure are in a monotone relation on $\Gamma_{\text {out }}$. Therefore they have their maxima at the same point $x_{M} \in$ $\bar{\Gamma}_{\text {out }}$,

$$
\max _{\bar{\Omega}} \bar{p}_{g}^{h}\left(\cdot, t_{M}\right)=\max _{\bar{\Gamma}_{\text {out }}} \bar{p}_{g}^{h}\left(\cdot, t_{M}\right)=\bar{p}_{g}^{h}\left(x_{M}, t_{M}\right) .
$$

Maximality of the functions together with Hopf's Lemma $\left(\bar{p}_{g}^{h}\left(\cdot, t_{M}\right)\right.$ satisfies an elliptic equation and is not constant on $\bar{\Omega}$ ) implies

$$
\begin{aligned}
n \cdot \nabla \bar{p}_{g}^{h}\left(x_{M}, t_{M}\right) & >0, \\
n \cdot \nabla\left(\bar{p}_{1}^{h}-\bar{p}_{2}^{h}\right)\left(x_{M}, t_{M}\right) & \geq 0 .
\end{aligned}
$$

Exploiting the definition of the global pressure and $k^{\delta} \partial_{s} p_{c}^{\delta} \nabla \bar{s}^{h} \cdot n=$ $k^{\delta} \nabla\left(\bar{p}_{1}^{h}-\bar{p}_{2}^{h}\right) \cdot n$ we find, with $1 \geq k_{\max }:=k^{\delta}(M)>0$,

$$
\begin{aligned}
0 & <n \cdot \nabla \bar{p}_{g}^{h}\left(x_{M}, t_{M}\right) \\
& =n \cdot \nabla \bar{p}_{2}^{h}\left(x_{M}, t_{M}\right)+k_{\max } n \cdot \nabla\left(\bar{p}_{1}^{h}-\bar{p}_{2}^{h}\right)\left(x_{M}, t_{M}\right) \\
& =n \cdot \nabla \bar{p}_{1}^{h}\left(x_{M}, t_{M}\right)-\left(1-k_{\max }\right) n \cdot \nabla\left(\bar{p}_{1}^{h}-\bar{p}_{2}^{h}\right)\left(x_{M}, t_{M}\right) \\
& \leq n \cdot \nabla \bar{p}_{1}^{h}\left(x_{M}, t_{M}\right) .
\end{aligned}
$$

This is in contradiction to the outflow boundary condition $n \cdot \nabla \bar{p}_{1}^{h} \leq 0$.

Lower bound for the saturation. In order to establish a lower bound for $\bar{s}^{h}$ we define

$$
p_{c, \min }:=\min _{\bar{\Gamma}_{D} \times[0, T]}\left(p_{1}^{D}-p_{2}^{D}\right),
$$

and

$$
s_{c, \text { min }}:=p_{c}^{-1}\left(p_{c, \min }\right), \quad s_{0, \min }:=\min _{\bar{\Omega}} s^{0} .
$$

Lemma 2.6. There exists $\delta_{1}>0$ such that, for all $\delta \leq \delta_{1}$, the saturation is bounded from below,

$$
\min _{\bar{\Omega}_{T}} \bar{s}^{h} \geq S_{\text {min }}:=\min \left(s_{\text {min }}, s_{0, \min }, a_{0}\right) .
$$


Proof. We choose $I \subset(a, b)$ with $s_{\min }, s_{0, \min }, a_{0} \in I$ and $\delta_{1}>0$ sufficiently small such that $p_{c}^{\delta}=p_{c}$ on $I$ for all $\delta \leq \delta_{1}$. For a contradiction we assume $m<S_{\min }$ such that $\bar{s}^{h}$ attains its minimal value $m$ in the point $\left(x_{m}, t_{m}\right)$ on the outflow boundary $\bar{\Gamma}_{\text {out }}$.

We first show that for $t=t_{m}$ the global pressure $\bar{p}_{g}^{h}=\bar{p}^{h}\left(\cdot, t_{m}\right)$ also has its minimum with respect to $\bar{\Omega}$ in $x_{m} \in \bar{\Gamma}_{\text {out }}$. By the definition of $\bar{p}_{g}^{h}$ and the assumption on the Dirichlet data we have

$$
\min _{\bar{\Gamma}_{D}} \bar{p}_{g}^{h} \geq \int_{a_{0}}^{s_{c, \text { min }}} k^{\delta} \partial_{s} p_{c}^{\delta}>\int_{a_{0}}^{m} k^{\delta} \partial_{s} p_{c}^{\delta}=\min _{\bar{\Gamma}_{\text {out }}} \bar{p}_{g}^{h}\left(\cdot, t_{m}\right)=\bar{p}_{g}^{h}\left(x_{m}, t_{m}\right) .
$$

By minimality of the saturation and Hopf's Lemma for the global pressure we find

$$
\begin{aligned}
n \cdot \nabla p_{g}^{h}\left(x_{m}, t_{m}\right) & <0, \\
n \cdot \nabla\left(\bar{p}_{1}^{h}-\bar{p}_{2}^{h}\right)\left(x_{m}, t_{m}\right) & \leq 0 .
\end{aligned}
$$

Therefore, with $0<k_{\min }:=k^{\delta}(m) \leq 1$,

$$
\begin{aligned}
0 & >n \cdot \nabla p_{g}^{h}\left(x_{m}, t_{m}\right) \\
& =n \cdot \nabla \bar{p}_{2}^{h}\left(x_{m}, t_{m}\right)+k_{m i n} n \cdot \nabla\left(\bar{p}_{1}^{h}-\bar{p}_{2}^{h}\right)\left(x_{m}, t_{m}\right) \\
& =n \cdot \nabla \bar{p}_{1}^{h}-\left(1-k_{\text {min }}\right) n \cdot \nabla\left(\bar{p}_{1}^{h}-\bar{p}_{2}^{h}\right)\left(x_{m}, t_{m}\right) \\
& \geq n \cdot \nabla \bar{p}_{1}^{h} .
\end{aligned}
$$

We find that outflow occurs in the minimum, the outflow condition implies $\bar{p}_{1}^{h}\left(x_{m}, t_{m}\right)=p_{c}^{\delta}(m)>0$. This is in contradiction with $m<S_{\min } \leq a_{0}$.

Bounds for the pressures. Since the capillary pressure $p_{c}$ is monotonically increasing, the bounds for the saturation imply

$$
p_{c}\left(S_{\text {min }}\right) \leq p_{c}^{\delta}\left(\bar{s}^{h}\right) \leq p_{c}\left(S_{\max }\right) \text { on } \bar{\Omega}_{T}
$$

for all $0<\delta \leq \min \left(\delta_{0}, \delta_{1}\right)$. We now show that the pressures $\bar{p}_{j}^{h}$ can be estimated with the help of the capillary pressure bounds.

Lemma 2.7. The following estimates hold for the pressures $\bar{p}_{j}^{h}, j=1,2$.

$$
\begin{aligned}
p_{c}\left(S_{\text {min }}\right) & \leq \bar{p}_{1}^{h} \leq p_{c}\left(S_{\text {max }}\right)+p_{2, \max }, \\
p_{c}\left(S_{\text {min }}\right)-p_{c}\left(S_{\text {max }}\right) & \leq \bar{p}_{2}^{h} \leq p_{c}\left(S_{\text {max }}\right)-p_{c}\left(S_{\text {min }}\right)+p_{2, \max }
\end{aligned}
$$

for all $0<\delta \leq \min \left(\delta_{0}, \delta_{1}\right)$. 
Proof. From Lemma 2.4 and the boundary condtions for $\bar{p}_{2}^{h}$ we obtain

$$
\bar{p}_{2}^{h}+\int_{a_{0}}^{\bar{s}^{h}} k^{\delta} \partial_{s} p_{c}=\bar{p}_{g}^{h} \leq p_{2, \max }+\int_{a_{0}}^{S_{\max }} k^{\delta} \partial_{s} p_{c}
$$

in $\bar{\Omega}_{T}$. Thus we get in $\bar{\Omega}_{T}$, since $k^{\delta} \leq 1$,

$$
\begin{aligned}
\bar{p}_{2}^{h} & \leq p_{2, \max }+\int_{\bar{s}^{h}}^{S_{\max }} k^{\delta} \partial_{s} p_{c} \leq p_{2, \max }+\int_{\bar{s}^{h}}^{S_{\max }} \partial_{s} p_{c} \\
& \leq p_{2, \max }+p_{c}\left(S_{\max }\right)-p_{c}\left(\bar{s}^{h}\right)
\end{aligned}
$$

Because of $\bar{s}^{h} \geq S_{\min }$ we have the second inequality of (2.24). Furthermore, we may insert $p_{c}\left(\bar{s}^{h}\right)=\bar{p}_{1}^{h}-\bar{p}_{2}^{h}$ on the right hand side. Then $\bar{p}_{2}^{h}$ cancels on both sides and we arrive at the upper bound of (2.23).

In the same way the lower bounds can be established. The maximum principle for $\bar{p}_{g}^{h}$ and $p_{2} \geq 0$ on $\Gamma_{0}$ provide

$$
\bar{p}_{2}^{h}+\int_{a_{0}}^{\bar{s}^{h}} k^{\delta} \partial_{s} p_{c}=\bar{p}_{g}^{h} \geq \int_{a_{0}}^{S_{\min }} k^{\delta} \partial_{s} p_{c},
$$

and therefore

$$
\bar{p}_{2}^{h} \geq \int_{\bar{s}^{h}}^{S_{\min }} k^{\delta} \partial_{s} p_{c} \geq p_{c}\left(S_{m i n}\right)-p_{c}\left(\bar{s}^{h}\right)
$$

in $\bar{\Omega}_{T}$. Monotonicity of $p_{c}$ and $\bar{s}^{h} \leq S_{\max }$ imply the first inequality in (2.24). Inserting $p_{c}\left(\bar{s}^{h}\right)=\bar{p}_{1}^{h}-\bar{p}_{2}^{h}$ yields the lower bound for $\bar{p}_{1}^{h}$ of (2.23).

Lemmas 2.5-2.7 imply the uniform bounds for solutions of (1.6)-(1.10). This concludes the proof of Theorem 1.

\section{Approximation result}

\subsection{Convergence and compactness}

Due to the maximum principle, the interpolations $\bar{s}^{h}$ and $\hat{s}^{h}$, respectively, are bounded away from the critical values where the coefficient functions degenerate. Therefore, the permeability functions $k_{j}^{\delta}$ are uniformly positive, $k_{j}^{\delta}\left(s^{\delta, h}\right) \geq c>0$ with a constant $c$ independent of $\delta$ and $h$, and the capillary pressure and its derivative remain bounded. We thus have uniform bounds for the saturations $\bar{s}^{h}$ and the pressures $\bar{p}_{j}^{h}$ in $L^{2}\left(0, T ; H^{1}(\Omega)\right), j=1,2$.

The following proposition summarizes the convergences which hold due to the a priori estimates and the boundedness of the regularized quantities. 
Proposition 3.1 (Convergences and limit functions). For every sequence $h \rightarrow 0$ and $\delta=\delta(h) \rightarrow 0$ there exists a subsequence $h \rightarrow 0$ and limit functions $s \in L^{\infty}\left(\Omega_{T}\right) \cap L^{2}\left(0, T ; H^{1}(\Omega)\right), p_{j} \in L^{2}\left(0, T ; H^{1}(\Omega)\right)$ and $v_{j} \in L^{2}\left(\Omega_{T}, \mathbb{R}^{n}\right)$ such that:

Convergence of the saturation.

$$
\begin{aligned}
& \hat{s}^{h} \rightarrow s \quad \text { and } \quad \bar{s}^{h} \rightarrow s \quad \text { in } L^{2}\left(\Omega_{T}\right), \\
& \bar{s}^{h} \rightarrow^{*} s \quad \text { in } L^{\infty}\left(\Omega_{T}\right), \quad \nabla \bar{s}^{h} \rightarrow \nabla s \quad \text { in } L^{2}\left(\Omega_{T}\right) .
\end{aligned}
$$

Convergence of the coefficient functions.

$$
\begin{aligned}
& k_{j}^{\delta}\left(\bar{s}^{h}\right) \rightarrow k_{j}(s), \quad p_{c}^{\delta}\left(\bar{s}^{h}\right) \rightarrow p_{c}(s) \quad \text { in } L^{2}\left(\Omega_{T}\right), \\
& P_{c}^{\delta}\left(\hat{s}^{h}\right) \rightarrow P_{c}(s)=\int_{a_{0}}^{s} p_{c}(\zeta) d \zeta \quad \text { in } L^{2}\left(\Omega_{T}\right) .
\end{aligned}
$$

Convergence of the pressures and velocities.

$$
\begin{aligned}
& \bar{p}_{j}^{h} \rightarrow p_{j} \quad \text { in } L^{2}\left(0, T ; H^{1}(\Omega)\right), \\
& \bar{v}_{j}^{h}=-k_{j}^{\delta}\left(\bar{s}^{h}\right) \nabla \bar{p}_{j}^{h} \rightarrow v_{j}=-k_{j}(s) \nabla p_{j} \quad \text { in } L^{2}\left(\Omega_{T}\right) .
\end{aligned}
$$

Proof. The most important result is the strong convergence of (3.1). Theorem 1 provides the non-degeneracy of the coefficients, thus the estimates (2.5) and (2.6) imply uniform bounds for

$$
\bar{s}^{h}, \bar{p}_{1}^{h}, \bar{p}_{2}^{h} \in L^{2}\left(0, T ; H^{1}(\Omega)\right) .
$$

The $L^{2} H^{1}$-norm of $\hat{s}^{h}$ is bounded by the $L^{2} H^{1}$-norm of $\bar{s}^{h}$. This fact, and the evolution equations (1.6)-(1.7) imply uniform bounds for

$$
\hat{s}^{h} \in L^{2}\left(0, T ; H^{1}(\Omega)\right), \quad \partial_{t} \hat{s}^{h} \in L^{2}\left(0, T ; H^{-1}(\Omega)\right) .
$$

We can apply the compactness theorem of Lions-Aubin (see e.g. [21]) and find the desired strong convergence of $\hat{s}^{h}$. Lemma 3.2 below provides the strong convergence of $\bar{s}^{h}$.

The weak convergences of (3.2), (3.5), and (3.6) follow from the uniform bounds for an appropriate subsequence.

The sequence $k_{j}^{\delta}$ converges uniformly to $k_{j}$ on $[0,1]$ by Assumption $2.1, \bar{s}^{h}$ converges strongly due to (3.1). This implies the first convergence of (3.3). For the second we exploit the uniform convergence of $p_{c}^{\delta} \rightarrow p_{c}$ on compact subsets of $(a, b)$ and the uniform bounds of Theorem 1 . The same facts imply (3.4). 
Lemma 3.2 (Comparison of interpolations). Let $X$ be a Hilbert space and $T>0$ a number, $h=T / N \rightarrow 0$ be a sequence with $N \in \mathbb{N}$. Let $t_{k}=h k$ and $f_{k}^{h} \in X$ for $k=0,1, \ldots, N$. We consider the piecewise affine interpolation $\hat{f}^{h}$ and the piecewise constant interpolation $\bar{f}^{h}$ of the point values $f_{k}^{h} \in X$,

$$
\begin{aligned}
\bar{f}^{h} \in V_{h} & :=\left\{\bar{f}^{h} \in L^{2}(0, T ; X) \mid \bar{f}^{h} \text { is constant on all intervals }\left(t_{k}, t_{k+1}\right]\right\}, \\
\hat{f}^{h} \in W_{h} & :=\left\{\hat{f}^{h} \in L^{2}(0, T ; X) \mid \hat{f}^{h} \text { is affine on all intervals }\left[t_{k}, t_{k+1}\right]\right\} .
\end{aligned}
$$

In this situation, the strong convergence

$$
\hat{f}^{h} \rightarrow g \text { in } L^{2}(0, T ; X)
$$

implies the convergences

$$
\begin{aligned}
\bar{f}^{h} & \rightarrow g \text { in } L^{2}(0, T ; X) \text { and } \\
h \partial_{t} \hat{f}^{h} & \rightarrow 0 \text { in } L^{2}(0, T ; X) .
\end{aligned}
$$

Proof. Step 1. We claim that

$$
\left\|\bar{f}^{h}\right\|_{L^{2}(0, T ; X)}^{2} \leq 6\left\|\hat{f}^{h}\right\|_{L^{2}(0, T ; X)}^{2}
$$

for all $\bar{f}^{h} \in V_{h}$ and $\hat{f}^{h} \in W_{h}$ that agree on the points $t_{k}$. The calculation is elementary,

$$
\begin{aligned}
& \left\|\hat{f}^{h}\right\|_{L^{2}((0, T), X)}^{2}=\sum_{k} h \int_{0}^{1}\left\|t f_{k}^{h}+(1-t) f_{k+1}^{h}\right\|_{X}^{2} \\
& \quad=\sum_{k} h \int_{0}^{1}\left(t^{2}\left\|f_{k}^{h}\right\|_{X}^{2}+(1-t)^{2}\left\|f_{k+1}^{h}\right\|_{X}^{2}\right)+2 h \int_{0}^{1} t(1-t)\left\langle f_{k}^{h}, f_{k+1}^{h}\right\rangle \\
& \quad=\sum_{k} \frac{h}{3}\left(\left\|f_{k}^{h}\right\|_{X}^{2}+\left\|f_{k+1}^{h}\right\|_{X}^{2}+\left\langle f_{k}^{h}, f_{k+1}^{h}\right\rangle\right) \\
& \quad \geq \frac{1}{6} \sum_{k} h\left(\left\|f_{k}^{h}\right\|_{X}^{2}+\left\|f_{k+1}^{h}\right\|_{X}^{2}\right) \geq \frac{1}{6} \int_{0}^{T}\left\|\bar{f}^{h}\right\|_{X}^{2} .
\end{aligned}
$$

Step 2. Given $g \in L^{2}(0, T ; X)$, we construct the averages

$$
g_{k}^{h}:=\frac{1}{h} \int_{t_{k-1}}^{t_{k}} g(t) d t
$$

and the corresponding piecewise affine and constant interpolations $\hat{g}^{h} \in W_{h}$ and $\bar{g}^{h} \in V_{h}$. We use the strong convergences of interpolations, $\hat{g}^{h} \rightarrow g$ and $\bar{g}^{h} \rightarrow g$ in $L^{2}(0, T ; X)$. By assumption,

$$
\left\|\hat{f}^{h}-\hat{g}^{h}\right\|_{L^{2}(0, T ; X)} \leq\left\|\hat{f}^{h}-g\right\|_{L^{2}(0, T ; X)}+\left\|g-\hat{g}^{h}\right\|_{L^{2}(0, T ; X)} \rightarrow 0 .
$$


Inequality (3.9) implies

$$
\left\|\bar{f}^{h}-\bar{g}^{h}\right\|_{L^{2}(0, T ; X)}^{2} \leq 6\left\|\hat{f}^{h}-\hat{g}^{h}\right\|_{L^{2}(0, T ; X)}^{2} \rightarrow 0 .
$$

Another application of the triangle inequality yields

$$
\left\|\bar{f}^{h}-g\right\|_{L^{2}(0, T ; X)} \leq\left\|\bar{f}^{h}-\bar{g}^{h}\right\|_{L^{2}(0, T ; X)}+\left\|\bar{g}^{h}-g\right\|_{L^{2}(0, T ; X)} \rightarrow 0 .
$$

Step 3. Convergence (3.8) follows from

$$
\begin{array}{r}
\left\|\bar{f}^{h}-\hat{f}^{h}\right\|_{L^{2}(0, T ; X)}^{2}=\sum_{k} h \int_{0}^{1}\left\|f_{k+1}^{h}-f_{k}^{h}\right\|_{X}^{2} t^{2} d t \\
=\sum_{k} \frac{h}{3}\left\|f_{k+1}^{h}-f_{k}^{h}\right\|_{X}^{2}=\frac{1}{3}\left\|h \partial_{t} \hat{f}^{h}\right\|_{L^{2}(0, T ; X)}^{2} .
\end{array}
$$

This concludes the proof.

\subsection{Equations for the limit functions}

We write (1.6) in the form

$$
\partial_{t} \hat{s}^{h}=\nabla \cdot\left(k_{1}^{\delta}\left(\bar{s}^{h}\right) \nabla \bar{p}_{1, k}^{h}\right) .
$$

Proposition 3.1 allows to take the limit and we find (1.13). The same argument yields (1.14). The constitutive relation (1.15) follows from the identity $p_{c}^{\delta}\left(\bar{s}^{h}\right)=\bar{p}_{1}^{h}-\bar{p}_{2}^{h}$ by passing to limits according to (3.3) and (3.5).

The limit saturation satisfies the relation $s(0)=s_{0}$ for $t=0$ in the weak sense. With the convergences of Proposition 3.1 we have for any $\phi \in$ $C_{0}^{\infty}(\Omega \times[0, T))$

$$
0=\int_{\Omega_{T}}\left(\hat{s}^{h} \partial_{t} \phi+\bar{v}_{1}^{h} \nabla \phi\right)+\int_{\Omega} s_{0} \phi(0) \rightarrow \int_{\Omega_{T}}\left(s \partial_{t} \phi+v_{1} \nabla \phi\right)+\int_{\Omega} s_{0} \phi(0) .
$$

On the Dirichlet boundary $\Gamma_{D}$ we can take the weak $L^{2}\left(\Gamma_{D, T}\right)$-limit

$$
0=\bar{p}_{j}^{h}-\bar{p}_{j}^{D, h} \rightarrow p_{j}-p_{j}^{D},
$$

due to the weak convergence of $\bar{p}_{j}^{h}$ and its trace. In the same way the Dirichlet condition for $p_{2}$ on the outflow boundary is obtained.

Concerning the Neumann condition we calculate, using the regularized equations with the notation $\hat{s}_{1}^{h}=\hat{s}^{h}, \hat{s}_{2}^{h}=1-\hat{s}^{h}$,

$$
0=\int_{\Gamma_{N}} \bar{v}_{j}^{h} \cdot n \phi=\int_{\Omega_{T}} \hat{s}_{j}^{h} \partial_{t} \phi+\int_{\Omega_{T}} \bar{v}_{j}^{h} \nabla \phi
$$

for any $\phi \in C_{0}^{\infty}\left(\left(\Omega \cup \Gamma_{N}\right) \times(0, T)\right)$. Passing to the limit on the right-hand side yields the corresponding expression for the limit functions which defines the normal velocity $v \cdot n$ on the boundary in distributional sense. 


\section{The outflow boundary condition}

For the proof of Theorem 2 it remains to verify the weak formulations of the outflow condition (1.4).

Regarding $v_{1} \cdot n \geq 0$ on $\Gamma_{\text {out }}$ we use an arbitrary function $\phi \in C_{0}^{\infty}((\Omega \cup$ $\left.\left.\Gamma_{\text {out }}\right) \times(0, T)\right)$ with $\phi \geq 0$ and calculate

$$
\begin{aligned}
0 & \leq \int_{\Gamma_{\text {out }, T}} \bar{v}_{1}^{h} \cdot n \phi=\int_{\Omega_{T}} \hat{s}^{h} \partial_{t} \phi+\int_{\Omega_{T}} \bar{v}_{1}^{h} \nabla \phi \\
& \rightarrow \int_{\Omega_{T}} s \partial_{t} \phi+\int_{\Omega_{T}} v_{1} \nabla \phi=\int_{\Gamma_{\text {out }, T}} v_{1} \cdot n \phi .
\end{aligned}
$$

Regarding $p_{1} \leq 0$ on $\Gamma_{\text {out }}$ we use $\phi \geq 0$ as above.

$$
\int_{\Gamma_{\text {out }, T}}\left(\bar{p}_{1}^{h}\right)_{+} \phi=-\int_{\Gamma_{\text {out }, T}} \delta \nabla \bar{p}_{1}^{h} \cdot n \phi=\delta \int_{\Gamma_{\text {out }, T}}\left[k_{1}^{\delta}\left(\bar{s}^{h}\right)\right]^{-1} \bar{v}_{1}^{h} \cdot n \phi .
$$

The saturations $\bar{s}^{h}$ are bounded away from the degeneracy of $k_{1}$, hence $\left[k_{1}^{\delta}\left(\bar{s}^{h}\right)\right]^{-1}$ is bounded by a constant $C_{k}$ independent of $\delta$ and $h$. Furthermore, the equality in (3.11) implies the boundedness of the outflow integral. We conclude, for $\phi \geq 0$,

$$
\int_{\Gamma_{\text {out }, T}} p_{1} \phi \leftarrow \int_{\Gamma_{\text {out }, T}} \bar{p}_{1}^{h} \phi \leq \int_{\Gamma_{\text {out }, T}}\left(\bar{p}_{1}^{h}\right)_{+} \phi \leq \delta C_{k} \int_{\Gamma_{\text {out }, T}} \bar{v}_{1}^{h} \cdot n \phi \leq C \delta(h) \rightarrow 0 .
$$

This yields $p_{1} \leq 0$ on $\Gamma_{\text {out }}$ in the sense of traces.

It remains to verify the variational inequality (1.16). Let $\phi_{j} \in C^{1}\left(\bar{\Omega}_{T}\right)$, $j=1,2$ be test functions, $\phi_{j}=p_{j}^{D}$ on $\Gamma_{D, T}$, with $\phi_{1} \leq 0$ and $\phi_{2}=0$ on $\Gamma_{\text {out }, T}$. We denote by $\bar{\phi}_{j}^{h}$ the piecewise constant interpolations and multiply (1.6) with $\left(\bar{\phi}_{1}^{h}-\bar{p}_{1}^{h}\right)$ and (1.7) with $\left(\bar{\phi}_{2}^{h}-\bar{p}_{2}^{h}\right)$. We obtain

$$
\begin{aligned}
\int_{\Omega_{t}} \partial_{t} \hat{s}^{h}\left(\bar{\phi}_{1}^{h}-\bar{p}_{1}^{h}\right)+\int_{\Omega_{t}} k_{1}^{\delta} \nabla \bar{p}_{1}^{h} \nabla\left(\bar{\phi}_{1}^{h}-\bar{p}_{1}^{h}\right) & =\int_{\Gamma_{\text {out }, t}} k_{1}^{\delta} \nabla \bar{p}_{1}^{h} \cdot n\left(\bar{\phi}_{1}^{h}-\bar{p}_{1}^{h}\right), \\
-\int_{\Omega_{t}} \partial_{t} \hat{s}^{h}\left(\bar{\phi}_{2}^{h}-\bar{p}_{2}^{h}\right)+\int_{\Omega_{t}} k_{2}^{\delta} \nabla \bar{p}_{2}^{h} \nabla\left(\bar{\phi}_{2}^{h}-\bar{p}_{2}^{h}\right) & =0 .
\end{aligned}
$$

Summing the equations and inserting the regularized boundary condition (1.9) yields

$$
\begin{aligned}
\int_{\Omega_{t}} \partial_{t} \hat{s}^{h}\left(\left(\bar{\phi}_{1}^{h}-\bar{p}_{1}^{h}\right)-\left(\bar{\phi}_{2}^{h}-\bar{p}_{2}^{h}\right)\right) & +\sum_{j=1}^{2} \int_{\Omega_{t}} k_{j}^{\delta}\left(\bar{s}^{h}\right) \nabla \bar{p}_{j}^{h} \nabla\left(\bar{\phi}_{j}^{h}-\bar{p}_{j}^{h}\right) \\
= & -\int_{\Gamma_{\text {out }, t}} k_{j}^{\delta}\left(\bar{s}^{h}\right) \frac{1}{\delta}\left(\bar{p}_{1}^{h}\right)_{+}\left(\bar{\phi}_{1}^{h}-\bar{p}_{1}^{h}\right) \geq 0
\end{aligned}
$$


where the inequality follows with a distinction of two cases, exploiting $\phi_{1} \leq 0$ on $\Gamma_{\text {out }, t}$. Using $\bar{p}_{1}^{h}-\bar{p}_{2}^{h}=p_{c}^{\delta}\left(\bar{s}^{h}\right)$ we define

$$
\begin{aligned}
I_{1}^{\delta, h} & :=\int_{\Omega_{t}} \partial_{t} \hat{s}^{h}\left(\bar{\phi}_{1}^{h}-\bar{\phi}_{2}^{h}\right), \quad I_{2}^{\delta, h}:=-\int_{\Omega_{t}} \partial_{t} \hat{s}^{h} p_{c}^{\delta}\left(\bar{s}^{h}\right), \\
I_{3}^{\delta, h} & :=\sum_{j=1}^{2} \int_{\Omega_{t}} k_{j}^{\delta}\left(\bar{s}^{h}\right) \nabla \bar{p}_{j}^{h} \nabla\left(\bar{\phi}_{j}^{h}-\bar{p}_{j}^{h}\right),
\end{aligned}
$$

and have $I_{1}^{\delta, h}+I_{2}^{\delta, h}+I_{3}^{\delta, h} \geq 0$. It remains to pass to the limit in each term. Convergence of $I_{1}^{\delta, h}$. With $\psi:=\phi_{1}-\phi_{2}$ we write

$$
I_{1}^{\delta, h}=\int_{\Omega_{t}} \partial_{t} \hat{s}^{h}\left(\bar{\psi}^{h}-\psi\right)+\int_{\Omega_{t}} \partial_{t} \hat{s}^{h} \psi
$$

In the first term we exploit $\left\|\bar{\psi}^{h}-\psi\right\|_{L^{2}\left(\Omega_{T}\right)}=O(h)$ and $h \partial_{t} \hat{s}^{h} \rightarrow 0$ in $L^{2}\left(\Omega_{T}\right)$ for $h \rightarrow 0$. The latter follows from (3.8) of Lemma 3.2. In the second integral we integrate by parts to obtain

$$
\int_{\Omega_{t}} \partial_{t} \hat{s}^{h} \psi=-\int_{\Omega_{t}} \hat{s}^{h} \partial_{t} \psi+\int_{\Omega} \hat{s}^{h}(t) \psi(t)-\int_{\Omega} s_{0} \psi(0) .
$$

By the strong convergence of $\hat{s}^{h}$ we have, for almost every $t$

$$
\lim _{h \rightarrow 0} I_{1}^{\delta, h}=-\int_{\Omega_{t}} s \partial_{t} \psi+\int_{\Omega} s(t) \psi(t)-\int_{\Omega} s_{0} \psi(0) .
$$

Convergence of $I_{2}^{\delta, h}$. We insert the term $p_{c}^{\delta}\left(\hat{s}^{h}\right)$ such that

$$
\begin{aligned}
I_{2}^{\delta, h} & =-\int_{\Omega_{t}} \partial_{t} \hat{s}^{h} p_{c}^{\delta}\left(\hat{s}^{h}\right)-\int_{\Omega_{t}} \partial_{t} \hat{s}^{h}\left(p_{c}^{\delta}\left(\bar{s}^{h}\right)-p_{c}^{\delta}\left(\hat{s}^{h}\right)\right) \\
& \leq-\int_{\Omega}\left(P_{c}^{\delta}\left(\hat{s}^{h}(t)\right)-P_{c}^{\delta}\left(s_{0}\right)\right),
\end{aligned}
$$

since the second integral is non-negative due to the monotonicity of the capillary pressure. The convergence (3.4) yields, for a.e. $t$,

$$
\liminf _{h \rightarrow 0} I_{2}^{\delta, h} \leq-\int_{\Omega}\left(P_{c}(s(t))-P_{c}\left(s_{0}\right)\right) .
$$

Convergence of $I_{3}^{\delta, h}$. We have

$$
I_{3}^{\delta, h}=\sum_{j=1}^{2} \int_{\Omega_{t}} k_{j}^{\delta}\left(\bar{s}^{h}\right) \nabla \bar{p}_{j}^{h} \nabla \bar{\phi}_{j}^{h}-\int_{\Omega_{t}} k_{j}^{\delta}\left(\bar{s}^{h}\right)\left|\nabla \bar{p}_{j}^{h}\right|^{2},
$$


where we can directly pass to the limit in the first integral by (3.3) and (3.5). The convergence $\left(k_{j}^{\delta}\left(\bar{s}^{h}\right)\right)^{\frac{1}{2}} \nabla \bar{p}_{j}^{h} \rightarrow\left(k_{j}(s)\right)^{\frac{1}{2}} \nabla p_{j}$ in $L^{2}\left(\Omega_{T}\right)$ and the weak lower semicontinuity of the $L^{2}\left(\Omega_{T}\right)$-norm implies

$$
\liminf _{h \rightarrow 0} I_{3}^{\delta, h} \leq \sum_{j=1}^{2} \int_{\Omega_{t}} k_{j}(s) \nabla p_{j} \nabla \phi_{j}-\int_{\Omega_{t}} k_{j}(s)\left|\nabla p_{j}\right|^{2} .
$$

The relation

$$
0 \leq \liminf _{h \rightarrow 0}\left(I_{1}^{\delta, h}+I_{2}^{\delta, h}+I_{3}^{\delta, h}\right)
$$

together with the expressions in (3.13)-(3.15) provide the variational inequality (1.16). This concludes the proof of Theorem 2.

\section{Further remarks and conclusions.}

Separate limits. Our approach allows any combined convergence $(h, \delta) \rightarrow$ 0 . It is also interesting to study the limit $h \rightarrow 0$ with $\delta>0$ fixed, i.e. to search for time-continuous solutions of the $\delta$-regularized problem. We remark that even in the case of a regularized outflow condition, the validity of the boundary condition in the limit $h \rightarrow 0$ is not clear. The same problem appears in the limit $\delta \rightarrow 0$ with $h>0$ fixed.

Bounded capillary pressure. We have assumed that the $p_{c}$-curve is unbounded at $a$ and at $b$, but the opposite case is also interesting and relevant in applications. In the case of a bounded $p_{c}$-function one may approximate the $p_{c}$-curve with a sequence of unbounded functions $p_{c}^{\delta}$. Our a priori estimates can be applied to the approximate problems, but the limit procedure becomes intricate due to the degenerate coefficient functions.

Conclusions. In this contribution we have derived uniform bounds for solutions of the two-phase flow system with an outflow condition. At first sight, one may think that such estimates follow easily from the parabolic character of the equations. Nonetheless, they are hard to prove since neither $p_{1}$ nor $p_{2}$ satisfies a maximum principle, and the global pressure satisfies no boundary condition on the outflow boundary.

We used the uniform estimates to study initial saturations that are not in the degenerate regime. In this case, the equations remain non-degenerate for all times and we find solutions of the original outflow problem.

Acknowledgment. The authors gratefully acknowledge the support by the Swiss grant SNF 03JANCHD and the German grant BMBF 03SF0310B. 


\section{References}

[1] H.W. Alt and E. DiBenedetto. Nonsteady flow of water and oil through inhomogeneous porous media. Ann. Scuola Norm. Sup. Pisa Cl. Sci. (4), 12(3):335-392, 1985.

[2] H.W. Alt, S. Luckhaus, and A. Visintin. On nonstationary flow through porous media. Ann. Mat. Pura Appl. (4), 136:303-316, 1984.

[3] T. Arbogast. The existence of weak solutions to single porosity and simple dual-porosity models of two-phase incompressible flow. Nonlinear Anal., 19(11):1009-1031, 1992.

[4] A. Beliaev. Homogenization of two-phase flows in porous media with hysteresis in the capillary relation. European J. Appl. Math., 14(1):6184, 2003.

[5] A. Yu. Beliaev and S. M. Hassanizadeh. A theoretical model of hysteresis and dynamic effects in the capillary relation for two-phase flow in porous media. Transp. Porous Media, 43(3):487-510, 2001.

[6] A. Bourgeat, S. Luckhaus, and A. Mikelić. Convergence of the homogenization process for a double-porosity model of immiscible two-phase flow. SIAM J. Math. Anal., 27(6):1520-1543, 1996.

[7] Z. Chen. Degenerate two-phase incompressible flow. I. Existence, uniqueness and regularity of a weak solution. J. Differential Equations, 171(2):203-232, 2001.

[8] Z. Chen. Degenerate two-phase incompressible flow. II. Regularity, stabiltiy and stabilization. J. Differential Equations, 186(2):345-376, 2002.

[9] D. Kröner and S. Luckhaus. Flow of oil and water in a porous medium. J. Differential Equations, 55(2):276-288, 1984.

[10] O.A. Ladyzenskaja and N.N. Uralceva. Linear and quasilinear elliptic equations, volume 46 of Mathematics in science and engineering. Academic Press, 1968.

[11] A. Michel. A finite volume scheme for two-phase immiscible flow in porous media. SIAM J. Numer. Anal., 41(4):1301-1317 (electronic), 2003.

[12] J.H. Nam and M. Kaviany. Effective diffusivity and water-saturation distribution in single- and two-layer PEMFC diffusion medium. International Journal of Heat and Mass Transfer, 46:4595-4611, 2003. 
[13] M. Ohlberger. Convergence of a mixed finite elements-finite volume method for the two phase flow in porous media. East-West J. Numer. Math., 5(3):183-210, 1997.

[14] M. Ohlberger and B. Schweizer. Modelling of interfaces in unsaturated porous media. In Discrete and continuous dynamical systems, Supplement Volume. Conference Proceedings of the AIMS, www.aimSciences.org, 2007.

[15] U. Pasaogullari and C.Y. Wang. Liquid water transport in gas diffusion layer of polymer electrolyte fuel cells. Journal of the Electrochemical Society, 151(3):A399-A406, 2004.

[16] R. Herbin R. Eymard and A. Michel. Mathematical study of a petroleum-engineering scheme. ESAIM: M2AN, 37(6):937-972, 2003.

[17] B. Schweizer. A well-posed model for dynamic contact angles. Nonlinear Anal., 43(1):109-125, 2001.

[18] B. Schweizer. Averaging of flows with capillary hysteresis in stochastic porous media. European J. Appl. Math., 18(3):389-415, 2007.

[19] B. Schweizer. Regularization of outflow problems in unsaturated porous media with dry regions. J. Differential Equations, 237:278-306, 2007.

[20] B. Schweizer. Homogenization of degenerate two-phase flow equations with oil trapping. SIAM J. Math. Anal., 39(6):1740-1763, 2008.

[21] R. E. Showalter. Monotone operators in Banach space and nonlinear partial differential equations, volume 49 of Mathematical Surveys and Monographs. American Mathematical Society, Providence, RI, 1997. 


\section{Preprints ab 2008}

2008-01

2008-02

2008-03

2008-04

2008-05

2008-06

2008-07

2008-08

2008-09

$2008-10$

2008-11

$2008-12$
Henryk Zähle

Weak approximation of SDEs by discrete-time processes

\section{Benjamin Fine, Gerhard Rosenberger}

An Epic Drama: The Development of the Prime Number Theorem

Benjamin Fine, Miriam Hahn, Alexander Hulpke, Volkmar große Rebel, Gerhard Rosenberger, Martin Scheer

All Finite Generalized Tetrahedron Groups

Ben Schweizer

Homogenization of the Prager model in one-dimensional plasticity

Benjamin Fine, Alexei Myasnikov, Gerhard Rosenberger

Generic Subgroups of Group Amalgams

Flavius Guiaş

Generalized Becker-Döring Equations Modeling the Time Evolution of a Process of Preferential Attachment with Fitness

Karl Friedrich Siburg, Pavel A. Stoimenov

A scalar product for copulas

Karl Friedrich Siburg, Pavel A. Stoimenov

A measure of mutual complete dependence

Karl Friedrich Siburg, Pavel A. Stoimenov

Gluing copulas

Peter Becker-Kern, Wilfried Hazod

Mehler hemigroups and embedding of discrete skew convolution semigroups on simply connected nilpotent Lie groups

Karl Friedrich Siburg

Geometric proofs of the two-dimensional Borsuk-Ulam theorem

Michael Lenzinger and Ben Schweizer

Two-phase flow equations with outflow boundary conditions in the hydrophobic-hydrophilic case 